\title{
A Simple and Effective Real-Time Controller for Wave Energy Converters
}

\author{
Francesco Fusco, Student Member, IEEE, and John V. Ringwood, Senior Member, IEEE
}

\begin{abstract}
A novel strategy for the real-time control of oscillating wave energy converters (WECs) is proposed. The controller tunes the oscillation of the system such that it is always in phase with the wave excitation force and the amplitude of the oscillation is within given constraints. Based on a nonstationary, harmonic approximation of the wave excitation force, the controller is easily tuned in real-time for performance and constraints handling, through one single parameter of direct physical meaning. The effectiveness of the proposed solution is assessed for a heaving system in one degree of freedom, in a variety of irregular (simulated and real) wave conditions. A performance close to reactive control and to model predictive control is achieved. Additional benefits in terms of simplicity and robustness are obtained.
\end{abstract}

Index Terms-Optimal control, real-time estimation, wave energy, wave forecasting.

\section{NOMENCLATURE}

$\begin{array}{ll}z(t), Z(\omega) & \text { Fourier transform pair. } \\ \hat{z} & \text { Complex amplitude of } z(t)=\Re\left\{\hat{z} e^{\jmath \omega t}\right\} . \\ x(t) & \text { Heaving position of floating body. } \\ v(t) & \text { Heaving velocity of floating body. } \\ f_{\mathrm{ex}}(t) & \text { Wave excitation force. } \\ f_{u}(t) & \text { Power takeoff force (control input). } \\ Z_{i}(\omega) & \text { Intrinsic impedance of floating system. } \\ Z_{r}(\omega) & \text { Radiation impedance of floating system. } \\ B(\omega) & \text { Radiation resistance of floating system. } \\ 1 / H & \text { Ratio velocity to excitation force. } \\ K(s) & \text { Feedforward controller for velocity tracking. } \\ H_{s} & \text { Significant wave height. } \\ \omega_{0} & \text { Peak frequency. } \\ \lambda & \text { Sharpness of Ochi standard spectrum. } \\ \Delta \omega & \text { Peaks spacing in double-peaked spectrum. }\end{array}$

Manuscript received October 10, 2011; revised April 19, 2012; accepted April 20, 2012. Date of publication May 25, 2012; date of current version December 12, 2012. This work was funded by the Irish Research Council of Science, Engineering and Technologies (IRCSET) under the Embark Initiative.

F. Fusco was with the Centre for Ocean Energy Research, National University of Ireland Maynooth, Maynooth, County Kildare, Ireland. He is now with the IBM Smart City Research Centre of Dublin, Dublin, Ireland (e-mail: francfus@ie.ibm.com).

J. V. Ringwood is with the Centre for Ocean Energy Research, National University of Ireland Maynooth, Maynooth, County Kildare, Ireland (e-mail: john.ringwood@eeng.nuim.ie).

Color versions of one or more of the figures in this paper are available online at http://ieeexplore.ieee.org.

Digital Object Identifier 10.1109/TSTE.2012.2196717

\author{
PTO Power takeoff. \\ $\mathrm{RCW} \quad$ Relative capture width.
}

\section{INTRODUCTION}

$\mathbf{O}$ CEAN wave power represents a resource which can make a significant contribution to the development of a more sustainable, and renewable, energy strategy for several countries worldwide [1]-[3]. The economic performance of WECs, however, is still far from competitive and significant scope exists for the improvement of the capacity factor, through more intelligent control systems. In particular, the efficiency of oscillating WECs can be significantly increased through an automatic control that tunes its oscillations to the incident wave elevation, in such a way as to improve the power transfer from the ocean to the system.

Control strategies for wave energy conversion are usually based on complex-conjugate control, which gives the optimal conditions, in the frequency domain, for maximum wave energy absorption [4], [5]. A real-time implementation of complex-conjugate control, however, is not practical, due to its noncausality [4], [6] and to the fact that physical constraints are not taken into account. Alternative control solutions have also been proposed, where the limitation imposed by the physics of the system (e.g., amplitude of motion or velocity, applicable forces) are explicitly accounted for. In particular, some of these alternatives include latching [7]-[9], where the oscillation in the system is delayed so to be in phase with the excitation from the waves, and model predictive control (MPC) [10]-[13], which is based on a constrained optimization. Controllers that adjust the damping and stiffness coefficients of the power takeoff (PTO) mechanism, in real-time, were also proposed. In [14], a fuzzylogic controller tunes the damping based also on predictions of the incident wave elevation. However, results are not compared with latching or MPC types of controllers and the assumption of linear damping can be unrealistic with most PTOs (hydraulic in particular).

While, in theory, latching allows the achievement of high energy capture, its applicability has been questioned [15], due the excessive loads on the latching mechanism, the disappointing performance in the case of self-reacting point absorbers [16], and the long (some minutes) prediction horizon required for the real-time optimization in irregular waves to converge [7], [9]. Solutions based on MPC, on the other hand, have shown many benefits, including the ability to handle constraints in a straightforward way [12], [13]. Such techniques, however, need to solve a constrained optimization problem at each time-step, that can be time-consuming if a complex model of the WEC 
is utilized. Besides, the performance of the controller depends on a variety of variables (cost function, optimization algorithm, future horizon, specification of the constraints, etc.) that are not always related to the physical domain of the problem and can only be tuned by extensive simulation. MPC-based controllers also require prediction of the excitation force which, although possible for 1-2 wave periods [17], introduces additional uncertainty in the solution.

This paper proposes an alternative controller that, while being suboptimal, is simple and effective at the same time, which makes it well suitable for real-time applications. In particular, the proposed controller has a hierarchical structure where a reference velocity is calculated from the current value of the wave force through a constant of proportion, which is tunable in real-time for performance optimization and constraints handling. The reference velocity is then imposed on the system by a low-level control loop based on classical control theory. Based on a nonstationary, harmonic model of the excitation force, the reference generation does not require an optimization, like MPC, and does not rely heavily on the model, which makes it inherently more robust to model uncertainties, through an appropriate design of the low-level controller.

The controller is a reactive type and it requires a PTO capable of implementing a bidirectional energy flow. Typical hydraulic PTOs and linear electrical generators that have been studied for applications in wave energy have the ability of inverting the energy flow [18], [19]. The nonideal efficiency, however, needs to be taken into account for an appropriate evaluation of the performance of the device [20], [21]. While this paper assumes ideal efficiency, since the focus is on the controller's concept, a quantitative discussion about the requirement of active power is proposed in Section IV.

The controller is derived in Section III, following introduction of the model of the wave energy conversion system under study in Section II. Validation against ideal complex-conjugate control (in the unconstrained case) and MPC (in the constrained case) is documented in Section IV, under a variety of random waves. Conclusions are discussed in Section V.

\section{Model of WAVE Energy CONVERTER}

\section{A. Model Definition}

A single-body floating system, as in Fig. 1, is considered where energy is extracted from the relative motion with the sea bottom, through a generic PTO mechanism. The system is constrained to move in the heave direction only. The external forces acting on the WEC are the excitation from the waves and the control force produced by the PTO. Under the assumption of linearity, neglecting viscosity, and other losses, the force-velocity model can be expressed in the frequency domain, as follows [4]:

$$
\jmath \omega M V(\omega)+Z_{r}(\omega) V(\omega)+\frac{K_{s}}{\jmath \omega}=F_{\mathrm{ex}}(\omega)+F_{u}(\omega) .
$$

In (1), $V(\omega)$ is the heaving velocity, while $F_{\mathrm{ex}}(\omega)$ and $F_{u}(\omega)$ are the wave excitation and PTO forces. The imbalance between gravity and the hydrostatic restoring force is modeled by the

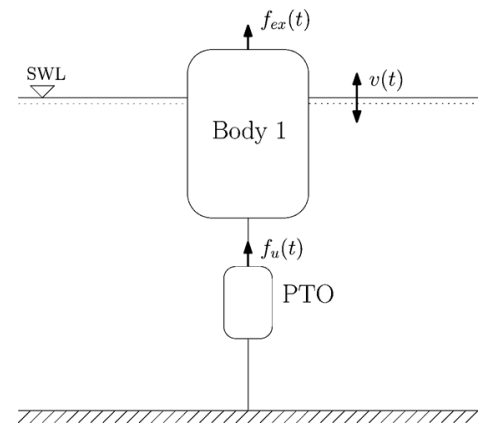

Fig. 1. One-degree-of-freedom (heave) floating system for wave energy conversion.

stiffness coefficient $K_{s}$. The radiation force, due to waves radiated by the body's motion, is expressed through the radiation impedance $Z_{r}(\omega)$. Notice that $Z_{r}(\omega)$ can only be considered as a Fourier transform in a generalized sense, as its imaginary part is unlimited for $\omega \rightarrow \infty$, so that it is often decomposed as

$$
\begin{aligned}
Z_{r}(\omega) & =B(\omega)+\jmath \omega\left[M_{a}(\omega)+M_{\infty}\right] \\
& =H_{r}(\omega)+\jmath \omega M_{\infty}
\end{aligned}
$$

where $B(\omega)$ is the radiation resistance (real and even) and $M_{a}(\omega)$ is the added mass after the singularity at infinite frequency $M_{\infty}$ is removed. The function $H_{r}(\omega)$ is a valid Fourier transform.

The model in (1) can be expressed in the compact form

$$
V(\omega)=\frac{1}{Z_{i}(\omega)}\left[F_{\mathrm{ex}}(\omega)+F_{u}(\omega)\right]
$$

where the intrinsic impedance $Z_{i}(\omega)$ is defined as

$$
Z_{i}(\omega)=B(\omega)+\jmath \omega\left[M+M_{a}(\omega)+M_{\infty}-\frac{K_{s}}{\omega^{2}}\right]
$$

The excitation force is the effect of the incident wave on the floating system

$$
F_{\mathrm{ex}}(\omega)=H_{\mathrm{ex}}(\omega) \Xi(\omega)
$$

where $\Xi(\omega)$ is the Fourier transform of the incident wave elevation $\eta(t)$. The transfer function $H_{\mathrm{ex}}(\omega)$ is a property of the WEC and has low-pass filter characteristics. Note, however, that $H_{\mathrm{ex}}(\omega)$ is noncausal, since the incident wave is not the cause of the force (the pressure distribution is) [4] and cannot be implemented in real-time simulations.

The example WEC system considered in this study consists of a heaving cylinder with radius $R=3 \mathrm{~m}$, height $H=5 \mathrm{~m}$, draught $h=4 \mathrm{~m}$, and mass $M=3.2 \times 10^{5} \mathrm{Kg}$. The radiation and excitation transfer functions, $H_{r}(\omega)$ and $H_{\mathrm{ex}}(\omega)$, are identified numerically through the hydrodynamic software Wamit [22] and are shown in Fig. 2.

\section{B. Model Identification}

The linear model in (3) is only known numerically for some frequencies. This is due to the fact that the radiation impedance, 

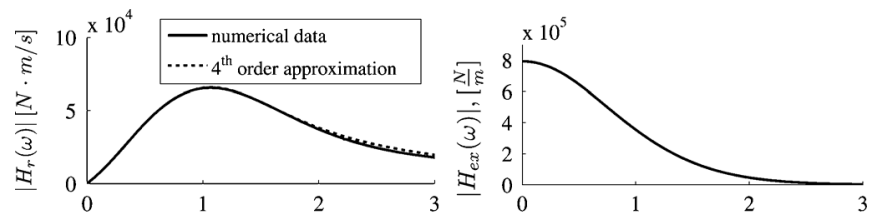

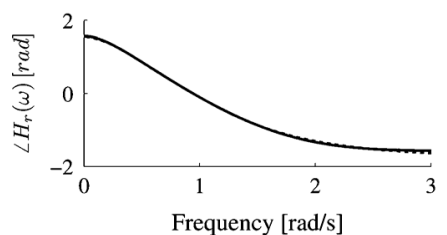

(a)

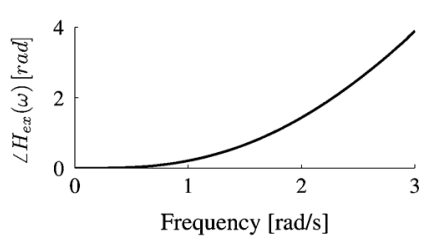

(b)
Fig. 2. Radiation and excitation frequency responses for a floating cylinder with radius $R=3 \mathrm{~m}$, height $H=5 \mathrm{~m}$, draught $h=4 \mathrm{~m}$, mass $M=3.2 \times$ $10^{5} \mathrm{Kg}$, calculated using the hydrodynamic software Wamit [22]. (a) Radiation. (b) Excitation.

$Z_{r}(\omega)$ or $H_{r}(\omega)$, can only be derived numerically from hydrodynamic simulation software or from experiments.

From a finite-order approximation of the radiation impedance, $\hat{H}_{r}(s) \approx H_{r}(\jmath \omega)$, the floating system in (3) can be approximately modeled as the following SISO system:

$$
\frac{V(s)}{F_{\mathrm{ex}}(s)+F_{u}(s)}=\frac{s}{s^{2}\left(m+M_{\infty}\right)+s \hat{H}_{r}(s)+K_{s}} \triangleq \frac{1}{Z_{i}(s)}
$$

where $s$ is the complex frequency in the Laplace domain. The input is the external force (from the waves or from the PTO), while the output is the system's velocity.

For the geometry specified in Fig. 2, a fourth-order approximation of the radiation was calculated using frequency-domain identification, following the approach in [23]. The result of the identification, shown in Fig. 2(a), gives a very accurate estimation of the radiation response of the system. A sixth-order forcevelocity system is, therefore, obtained from (6), and is specified by the following transfer function (coefficients rounded to one decimal place for brevity):

$$
\frac{1}{Z_{i}(s)}=\frac{1.8 \times 10^{-6} \cdot s\left(s^{2}+1.1 s+0.4\right)\left(s^{2}+1.6 s+1.8\right)}{\left(s^{2}+1.2 s+0.4\right)\left(s^{2}+1.4 s+1.6\right)\left(s^{2}+0.1 s+1.5\right)} \text {. }
$$

\section{REAL-Time CONTROLLER}

This section outlines the proposed strategy for real-time control of a WEC of the type modeled in Section II. As shown in Fig. 3, the approach consists of the generation of a reference velocity, from knowledge of the current wave excitation force acting on the system, and then imposition of such velocity, through the PTO force, via a lower-level feedback controller $K(s)$. After an analysis of the conditions for maximum wave energy absorption, in Section III-A, the reference-generation strategy is derived in Section III-B. Section III-C shows the possibility of handling constraints and Section III-D describes the design of $K(s)$.

\section{A. Maximum Wave Energy Extraction}

As extensively studied in [4], maximum energy transfer from the waves to the system PTO is obtained when

$$
F_{u}(\omega)=-Z_{i}^{*}(\omega) V(\omega)
$$

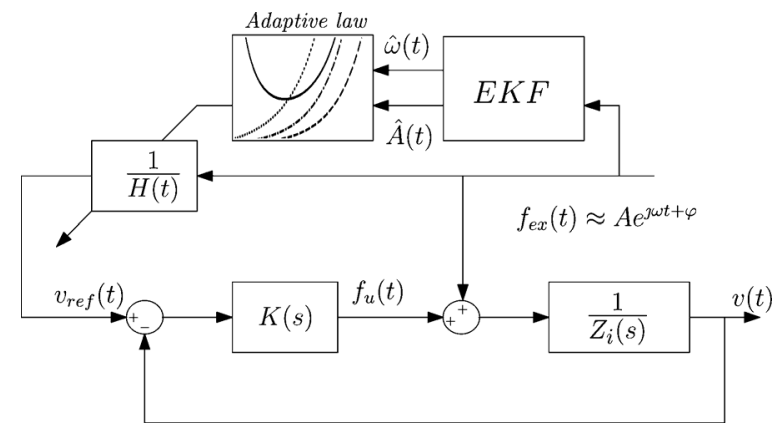

Fig. 3. Proposed control architecture.

where $Z_{i}^{*}(\omega)$ is the complex-conjugate of the intrinsic impedance of the system. The condition in (8), however, is anti-causal and cannot be implemented in real-time.

The control law in (8) is termed complex-conjugate control (note the electrical analogy from impedance matching) or reactive control, from the requirement of a bidirectional energy, which means that a negative energy absorption would be experienced for parts of the cycle.

An alternative, and equivalent, condition for maximum energy extraction [4] is a phase and amplitude relation between oscillation velocity and wave excitation force

$$
V(\omega)=\frac{1}{Z_{i}(\omega)+Z_{i}^{*}(\omega)} F_{\mathrm{ex}}(\omega)=\frac{1}{2 B(\omega)} F_{\mathrm{ex}}(\omega) .
$$

The radiation resistance $B(\omega)$ is a real and even function in the frequency domain. As a consequence, the phase and amplitude condition in (9) is noncausal, and, in particular, the corresponding time-domain impulse response function is also real and even [4].

Under conditions (8) or (9), the average absorbed power $P_{u}(\omega)$ is maximum and it is half the average excitation power $P_{\text {ex }}(\omega)$, the other half being lost in radiation $P_{r}(\omega)$ [4].

It has been shown [6], [17] that the noncausality can be addressed with simple regressive predictors of excitation force. However, there are two more characteristics of reactive control that make its practical implementation difficult (if not impossible) in some situations:

- The optimal velocity, position, and PTO force can have very large amplitudes, well beyond physical constraints, particularly in large waves and for small systems.

- Since the energy flow in the PTO is bidirectional, power needs to be injected back into the system for part of the cycle, in order to achieve the optimum. Inefficiencies in the PTO, when reversing the energy flow, may, therefore, undermine the efficiency of the solution.

In Sections III-B and III-C, a nonoptimal approximation of reactive control is proposed, where the noncausality and the constraints are overcome in a simple, but effective, way. Note that, in the following, we assume that the WEC, modeled in Section II, has a PTO machinery capable of implementing a reversible energy flow with ideal efficiency.

\section{B. Simple and Effective Realization of Reactive Control}

Based on (9), the velocity should always be in phase with the excitation force and it should have an amplitude that is modulated in the frequency-domain by the inverse of double the ra- 


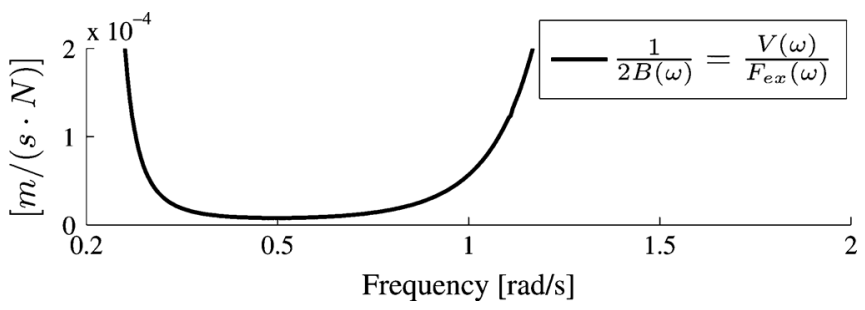

Fig. 4. Optimal relation between excitation force and oscillation velocity. The phase is zero.

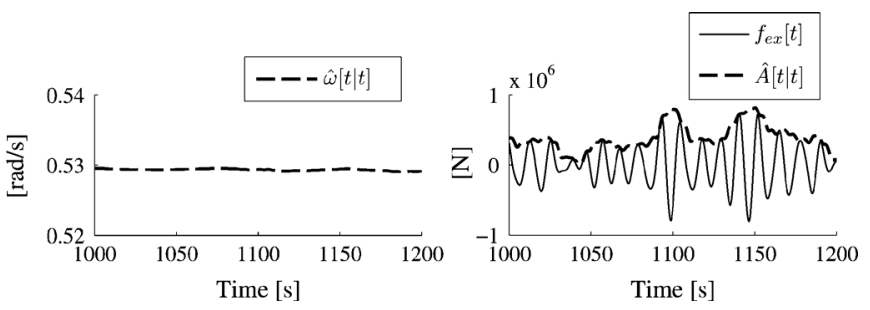

Fig. 5. Real-time estimation of amplitude and frequency of a stochastic excitation-force signal, generated from a power spectral distribution centered around $5.3 \mathrm{rad} / \mathrm{s}$.

diation resistance $1 / 2 B(\omega)$, which is noncausal. Predictions of the excitation force can be utilized to resolve the noncausality [6], [17]. This paper, however, proposes a nonoptimal simplification that does not require predictions.

The relation in (9) is a frequency-dependent gain, shown in Fig. 4, to be applied to the excitation force, such to obtain the velocity. The noncausality derives from the fact that, in theory, all the frequencies may be present at the same time. In practice, the excitation force is contained within a restricted band of frequencies, mostly within the flat part of $1 / 2 B(\omega)$. A constant approximation, based on a second-order model of the system, was already proposed in [24]. If a real-time estimate of the peak frequency of the excitation force is available, however, we can adapt the constant from the curve $1 / 2 B(\omega)$.

Assuming that the excitation, $f_{\mathrm{ex}}(t)$, is a narrowband harmonic process, defined by time-varying amplitude, $A(t)$, frequency, $\omega(t)$, and phase $\varphi(t)$

$$
f_{\mathrm{ex}}(t)=A(t) \cos (\omega(t) t+\varphi(t))
$$

the reference velocity can be generated from the following adaptive law:

$$
v_{\text {ref }}(t)=\frac{1}{H(t)} f_{\mathrm{ex}}(t), \quad \frac{1}{H(t)}=\frac{1}{2 B(\hat{\omega})}
$$

where the value of the constant $H(t)$ is calculated from the curve $1 / 2 B(\omega)$, based on a real-time estimate of the peak frequency of the wave excitation force.

An online estimate of the frequency $\hat{\omega}$ and amplitude $\hat{A}$ is obtained with the extended Kalman filter (EKF) [25], already proposed in [17], [26] for modeling the wave elevation. Although suboptimal, the estimation obtained with the EKF is satisfactory in our case, as shown in Fig. 5. Alternative methods that give a solution closer to optimal have also been proposed, as reviewed in [27] and [28].
Note that the sinusoidal assumption in (10) was considered by a number of researchers [29], [30], so to remove the noncausality of complex-conjugate control. However, simulations were always carried out in regular waves, so to satisfy the assumption. In the present paper, the controller is tested with irregular waves (simulated and real data), that do not necessarily satisfy the assumption, as discussed in Section IV.

\section{Constraints Handling}

The control solution proposed in Section III-B, where the velocity is simply calculated from the excitation force through a variable gain, allows the handling of physical constraints in a quite intuitive and effective way. Based on the narrowband assumption of (10), the excitation force can be expressed in complex notation as

$$
f_{\mathrm{ex}}(t)=\Re\left\{A e^{\jmath \varphi} e^{\jmath \omega t}\right\}, \quad \hat{F}_{\mathrm{ex}} \triangleq A e^{\jmath \varphi}
$$

where $\hat{F}_{\text {ex }}$ is termed complex amplitude of $f_{\text {ex }}(t)$.

As a consequence of the proportional reference-generation law, in (11), the complex amplitude of the velocity $\hat{V}$ and position $\hat{X}$ can be expressed as

$$
\begin{aligned}
& \hat{V}=\frac{A}{H} e^{\jmath \varphi} \\
& \hat{X}=\frac{\hat{V}}{\jmath \omega}=\frac{A}{\jmath \omega H} e^{\jmath \varphi} .
\end{aligned}
$$

Suppose that the vertical excursion of the WEC is limited to $\pm X_{\text {lim }} \mathrm{m}$ from equilibrium. From (14), the position constraint can be written as an equivalent velocity constraint

$$
\hat{X}=\frac{\hat{V}}{\jmath \omega} \leq X_{\lim } \Longleftrightarrow|\hat{V}| \leq \omega X_{\lim }
$$

and the following upper bound for the variable gain $1 / H$, involving the amplitude and frequency of the excitation, can be derived from (13)

$$
\frac{1}{H} \leq \frac{\omega X_{\lim }}{A}
$$

The reference generation strategy, based on (9), (11), and (16), can, therefore, be modulated in order to keep the amplitude of the velocity within the bound specified in (15). A real-time estimate of the frequency $\hat{\omega}$ and amplitude $\hat{A}$ of the excitation can be obtained through the EKF [17], [26]

$$
\frac{1}{H(t)}= \begin{cases}\frac{1}{2 B(\hat{\omega})}, & \text { if } \frac{\hat{\omega} X_{\lim }}{\hat{A}}>\frac{1}{2 B(\hat{\omega})} \\ \frac{\omega X_{\lim }}{\hat{A}}, & \text { otherwise. }\end{cases}
$$

According to (17), when in the unconstrained region, the velocity is tuned to the optimal amplitude given by complex-conjugate control, as in (9). Otherwise, the maximum allowed velocity (lower than the optimal) is imposed, while keeping the velocity in phase with the excitation force. Note that a velocity calculated from (17) is optimal within the proposed parametrization, which means that it gives the maximum energy extraction 


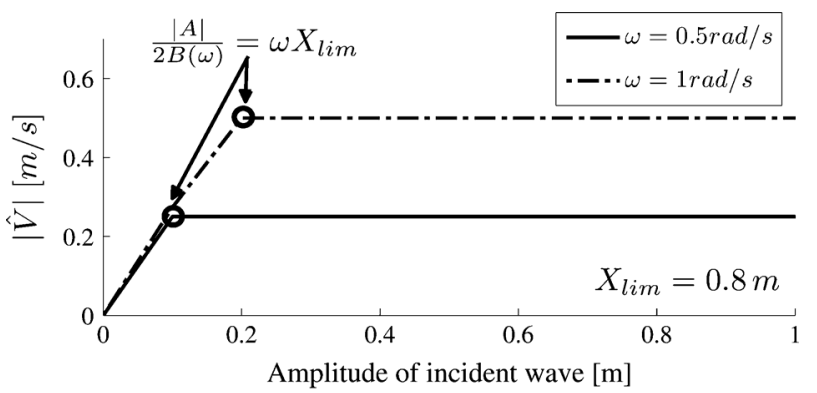

(a)

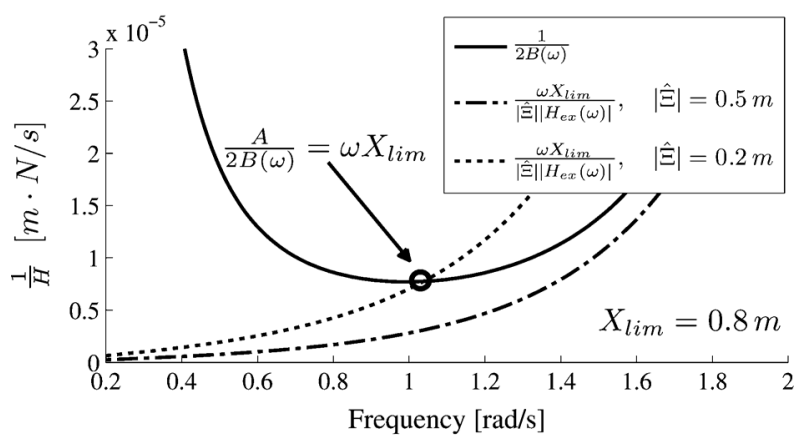

(b)

Fig. 6. Graphical representation of adaptive law (17). Note that magnitude of the excitation force $|\hat{A}|$ is translated in amplitude of the incident wave elevation, $\hat{\Xi}=\left|H_{\mathrm{ex}}(\omega) A\right|$, from (5). (a) Magnitude of velocity with respect to amplitude of incident wave elevation. (b) Ideal optimal value and upper limit, for a constraint $X_{\lim }=0.8 \mathrm{~m}$, of the adaptive constant $1 / H$, as from (17).

among all the velocities proportional to the excitation force and smaller than the constraint.

Given the geometry specified in Section II-A, and a constraint $X_{\text {lim }}=0.8 \mathrm{~m}$, Fig. 6(a) shows the profile of the reference velocity, for two frequencies, as a function of the amplitude of the incident wave elevation, which is related to the amplitude of the excitation force, $|\hat{\Xi}|=\left|A H_{\mathrm{ex}}(\omega)\right|$, from (5). For small waves, the velocity is modulated from the optimal condition given by reactive control, while above waves of a certain size it is kept at a constant maximum amplitude (smaller than optimal), so that constraints are satisfied. The limit of the unconstrained region depends on the frequency, as from Fig. 6(b). In the case of 0.2 -m waves, the controller would work at maximum allowed speed for $\omega<1 \mathrm{rad} / \mathrm{s}$, where $1 / 2 B(\omega)=\omega X_{\lim } / A$, while it would follow the optimal speed for higher frequencies. When the system is excited by $0.5-\mathrm{m}$ waves, the velocity is always tuned at the maximum allowed by the constraint (lower than the optimum).

Note that:

1) Ultimately, the controller is regulated only through a single parameter $1 / H$ that is the constant of proportionality between excitation force and velocity. Such a parameter can be tuned for best performance, as in (17), but also based on other requirements. It could, for example, be tuned for the regulation of the power at a desired level (absorbed power is proportional to the velocity squared).

2) Unlike optimization-based solutions, the proposed controller relies on the model of the system only in the unconstrained region. In the constrained region, the low-level control loop depends on the model, while the reference-generation does not, and a robust design would

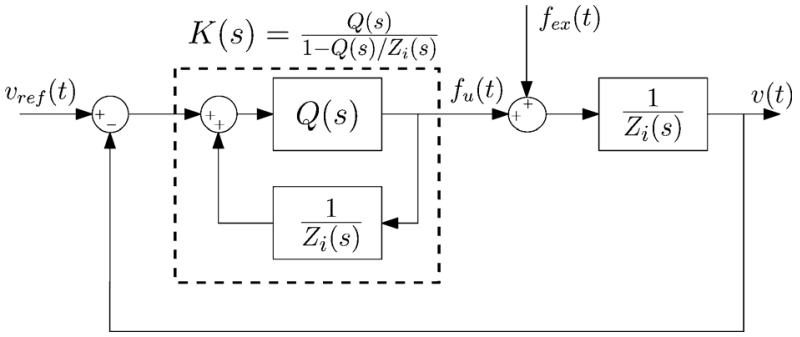

Fig. 7. Low-level controller based on IMC feedback structure.

benefit from this separation. Further discussion on improving the robustness will be mentioned in Section III-D.

3) Predictions of the excitation force are not necessary.

4) A practical implementation would only require an estimation of the excitation force, which is not a measurable quantity. An observer can be built from measurements of the motion (velocity, acceleration) of the system [10].

\section{Velocity-Tracking Control Loop}

The desired velocity, generated from (11), is imposed on the system by the low-level controller, that acts on the PTO force $f_{u}(t)$. The controller, namely $K(s)$, is based on the concept of internal model control (IMC) [31], that was also proposed for the control of the Archimedes Wave Swing WEC [32].

Fig. 7 shows the equivalent structure of the IMC. The compensator $K(s)$ is given by

$$
K(s)=\frac{Q(s)}{1-Q(s) / Z_{i}(s)}
$$

where $1 / Z_{i}(s)$ is the model of the system, given in (7), and $Q(s)$ is a filter that can be opportunely chosen. In the case of perfect model and no disturbances, it can be shown [31] that the controller works in open-loop and the feedforward compensator simplifies to the filter $Q(s)$. Feedback only appears in the presence of model uncertainties or disturbances.

As a consequence, $Q(s)$ can be intuitively designed for performance and disturbance rejection. In addition, it can be shown [31] that the stability of $Q(s)$ implies the stability of the closedloop system, at least in the case of perfect model.

As proposed in [31] and [32], the filter $Q(s)$ is designed as

$$
Q(s)=F(s) \cdot Z_{i}(s)
$$

where $Z_{i}(s)$ is the inverse of the system and $F(s)$ is a low-pass filter that ensures the stability and the physical realizability of $Q(s)$. Perfect control would require that $Q(s)=1$, but this is only achievable in a limited range of frequencies, due to the presence of $F(s)$.

From (6), it is derived that the model of the system $1 / Z_{i}(s)$ is proper with relative degree of 1 and is nonminimum phase due to the presence of a zero at $s=0$. Therefore, it is required that $F(s)$ has a zero at $s=0$, to cancel the unstable pole of $Z_{i}(s)$, and that it is proper with relative degree of 1 , for the physical realizability of $K(s)$.

In our specific case, the filter $F(s)$ is shaped as a bandpass filter

$$
F(s)=\frac{s}{(s+0.2)} \cdot \frac{5}{s+5}
$$




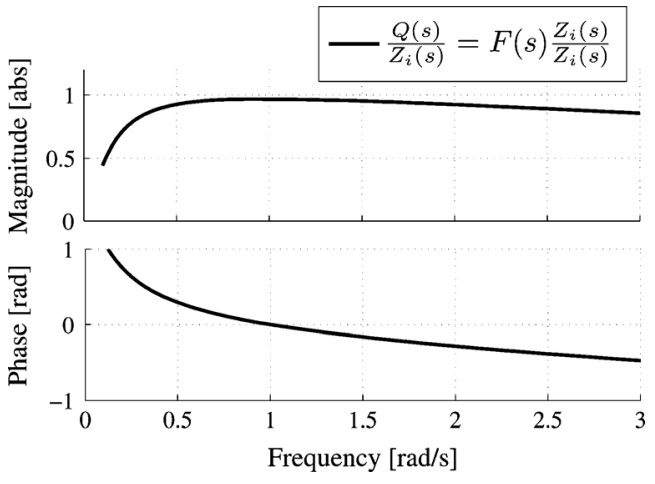

Fig. 8. Sensitivity function in the case of perfect model $Q(s) / Z_{i}(s)$.

where 0.2 and $5 \mathrm{rad} / \mathrm{s}$ are the cutoff frequencies of the high- and low-pass sections, respectively.

Fig. 8 shows that the sensitivity function of the controller in the case of perfect model approaches unity (good tracking) in the region $[0.3,2] \mathrm{rad} / \mathrm{s}$, which corresponds to the typical active band of frequencies of the excitation force. Note that the excitation force resulting from any possible sea state is always contained in such a region for the given system (due to the low-pass characteristics of the excitation filter $H_{\text {ex }}(\omega)$ given in [Fig. 2(b)] so that the controller $K(s)$ does not need to be adaptively tuned to the sea state, but its structure can be fixed at the design stage.

In the presence of model uncertainties, a procedure exists [31] to ensure robustness of the feedback controller. In order to maintain the focus on the performance of the reference-generation strategy, outlined in Section III-B, the robustness design is omitted.

\section{RESULTS}

\section{A. Wave Data}

The proposed control method is validated on the heaving cylinder as specified in Section II under several wave conditions. Random waves are generated from single- and doublepeaked standard wave spectral distributions. The three- and sixparameters Ochi spectral distributions [33] are utilized, which give the possibility of independently specifying the modal frequency $\omega_{0}$, the significant wave height $H_{s}$, and the sharpness $\lambda$

$S_{\eta \eta}(\omega)=\sum_{j=1,2} \frac{\left(\frac{4 \lambda_{j}+1}{4} \omega_{0, j}^{4}\right)^{\lambda_{j}}}{\Gamma\left(\lambda_{j}\right)} \cdot \frac{H_{s, j}^{2}}{\omega^{4 \lambda_{j}+1}} e^{-\left(4 \lambda_{j}+1 / 4\right)\left(\omega_{0, j} / \omega\right)^{4}}$

where $j=1,2$ are the components of the spectrum (only $j=1$ for the single-peak case) and $\Gamma(\cdot)$ is a Gamma function.

Fig. 9 shows some examples of the spectral distributions. The double-peak spectra are parameterized with the ratio $H_{s, 1} / H_{s, 2}$, that is the relative energy of the two peaks, and with the spacing between the peaks, $\Delta \omega=\omega_{0,2}-\omega_{0,1}$.

The variety of single-peak spectra has been chosen with $\omega_{0}$ ranging from 0.3 to $1.2 \mathrm{rad} / \mathrm{s}, \lambda$ from 0.5 to 5 (widebanded to narrowbanded spectrum), and $H_{s}=1$ or $H_{s}=3 \mathrm{~m}$. For the double-peaked spectra the ratio $H_{s, 1} / H_{s, 2}$ ranges from 4 to 0.5 ; the frequency spacing $\Delta \omega$ ranges from 0.2 to $0.9 \mathrm{rad} / \mathrm{s}$.

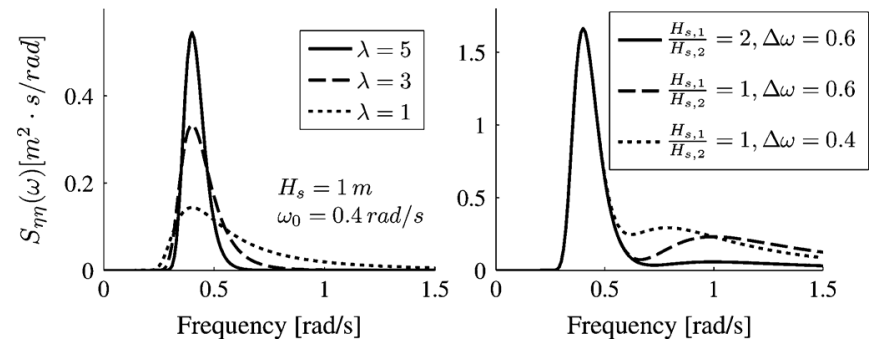

Fig. 9. Spectral shapes utilized for the simulation of the waves.

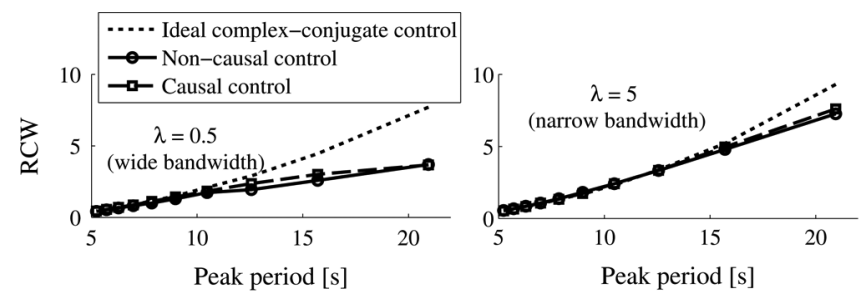

(a)
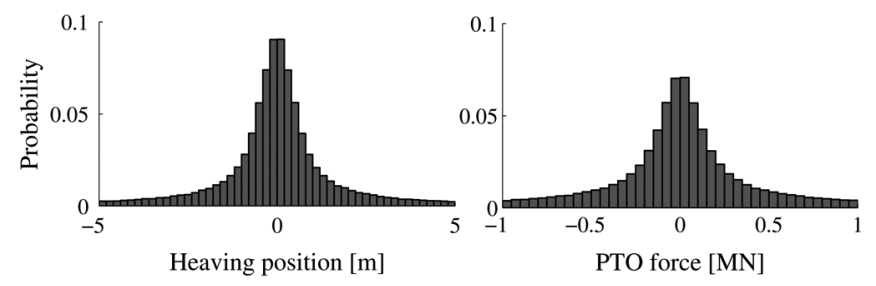

(b)

Fig. 10. Performance of proposed nonoptimal causal control. (a) RCW. (b) Distribution of heaving excursion and PTO force.

Wave time series of $3600 \mathrm{~s}$ sampled at $f_{s}=2.56 \mathrm{~Hz}$, are generated as superposition of harmonic signals

$$
\eta\left(k T_{s}\right)=\sum_{j=0}^{N / 2} a_{j} \sin \left(\omega_{j} k T_{s}+\phi_{j}\right)
$$

where the amplitudes $a_{j}$ are specified by the spectral shape $S_{\eta \eta}(\omega)$, the phases $\phi_{j}$ are randomly chosen from a uniform distribution in the interval $[0,2 \pi]$. The number of frequencies $N$, equally spaced in the interval $\left[0, \pi f_{s}\right]$, is chosen such that statistical properties of random waves are satisfied and that the signal $\eta(k)$ does not repeat itself within the simulation [34]. In particular, the signal $\eta(k)$ generated from (22) is periodic of $N \cdot T_{s} \mathrm{~s}$, so that it was chosen $N=3600 \cdot 2.56=9216$.

\section{B. Performance in the Unconstrained Case}

The performance of the controller proposed in Section III-B is initially validated for the unconstrained case. Complex-conjugate control, outlined in Section III-A, is utilized as a comparison. The controllers are implemented, as shown in Fig. 3, as a reference-velocity generator followed by a velocity-tracking loop, as designed in Section III-D. In the case of complex-conjugate control, the reference velocity is generated from the optimal noncausal law (9). The noncausality is resolved as proposed in [6], by assuming exact knowledge of the future excitation force, for the ideal case, and by using real-time predictions with an autoregressive (AR) model [17], labelled as noncausal controller in Fig. 10(a). 


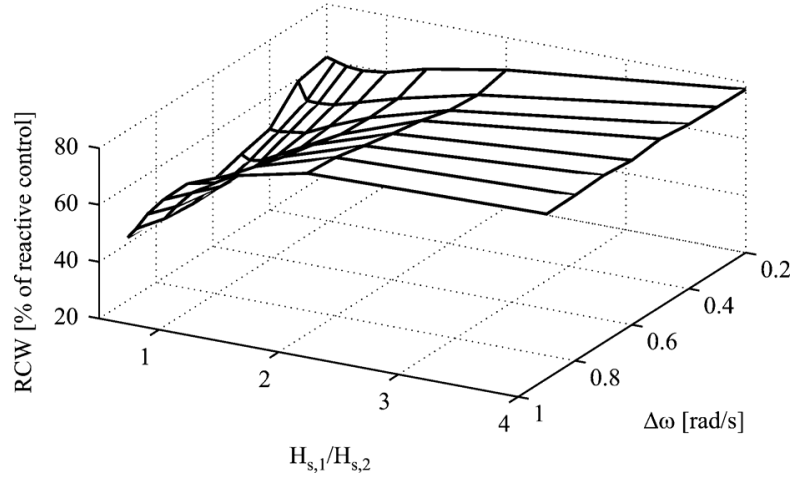

Fig. 11. Performance of causal control, as a percentage of ideal reactive control, with double peak spectra: $H_{s, 1} / H_{s, 2}$ is the relative energy between the two wave systems; $\Delta \omega$ is the frequency spacing.

Performance is measured in terms of relative capture width $(\mathrm{RCW})$

$$
\mathrm{RCW}=\frac{\bar{P}_{u}}{2 R P_{w}}
$$

which is the ratio between average absorbed power $\bar{P}_{u}$ and average wave power $P_{w}$ over a front as wide as the cylinder $(2 R)$. Note that deep water is assumed, so that the wave power, per meter of wave front, is calculated from the wave spectral distribution $S_{\eta \eta}(\omega)$ as [4]

$$
P_{w}=\frac{\rho g^{2}}{2} \int_{0}^{\infty} \frac{S_{\eta \eta}(\omega)}{\omega} d \omega \quad\left[\frac{W}{m}\right]
$$

where $\rho$ is the water density and $g$ is the acceleration due to gravity.

Fig. 10(a) shows the RCW obtained with the nonoptimal (and causal) controller compared to the ideal optimum, for widebanded $(\lambda=0.5)$ and narrowbanded $(\lambda=5)$ wave spectra, centered around different periods, $2 \pi / \omega_{0}$. The proposed controller performs very close to optimum, particularly for narrowbanded waves. For widebanded sea states, the performance is very close to the ideal maximum only for short to medium waves, while it significantly drops for very long waves. Note that only a minor improvement in performance is obtained with a noncausal approximation of complex-conjugate control, using predictions.

Note that RCW is quite large in most situations, and this is due to the fact that physical constraints are not taken into account, which is one of the limitations of ideal reactive control. Fig. 10(b) shows the distribution of the heaving position and PTO force required to obtain the results of Fig. 10(a). The position typically ranges from -5 to $5 \mathrm{~m}$ (with bigger excursion in some situations!), which is quite impractical for a floating cylinder $5 \mathrm{~m}$ high and with a 4-m draught. Also, PTO forces of more than $1 \times 10^{6} \mathrm{~N}$ are experienced in some occasions, which can require large and expensive machinery.

The performance of the proposed controller in double-peaked seas is also assessed.Fig. 11 shows the behavior of the RCW, relative to ideal optimum from reactive control. Clearly, the performance is negatively affected (below $50 \%$ of optimum) when the two wave systems have a similar energy content and when they are widely spaced.

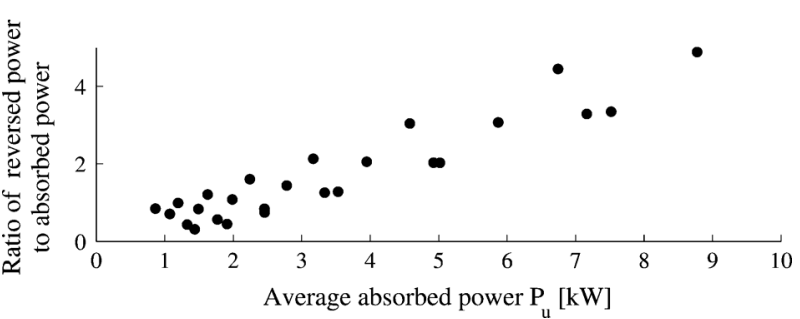

Fig. 12. Ratio of average reversed power (energy from PTO to system) to absorbed power (energy from system to PTO).

A final analysis is carried out in order to determine the amount of energy that needs to be injected from the PTO back into the system, during part of the cycle, known to be an issue with complex-conjugate control.Fig. 12 shows the ratio of the average reversed power, that is the average absorbed power of negative sign, to the overall average absorbed power (that is always positive if the controller is effective). Most of the time, a higher reversed energy flow than what is actually absorbed is required (between 1 and 5 times!). The ratio seems to increase with the average power absorption of the system but, in general, it looks quite unreasonable to achieve since it would require an excessive over-specification of the PTO.

High levels of reversed power, together with the excessive motion required, make unconstrained complex-conjugate control unpractical most of the time, apart in the case of very small waves (how small depends on the geometry and rating of the specific WEC).

\section{Introduction of Constraints}

The proposed causal and nonoptimal controller is also validated when physical constraints are imposed, as described in Section III-C. MPC, which solves a constrained optimization problem at each time-step, is utilized as a comparison, following the approach proposed in [12]. In particular, the future horizon for the optimization is set to 30 steps $(\approx 11.77 \mathrm{~s})$, which is about double the resonance period of the floating cylinder $(\approx 5.2 \mathrm{~s})$. For a fair comparison, predictions are not assumed to be ideal and a forecasting algorithm, based on AR models, is implemented, as proposed in [17].

Fig. 13 shows the RCW obtained from the two controllers, in wide- $(\lambda=0.5)$ and narrowbanded $(\lambda=5)$ sea states for different peak periods, $2 \pi / \omega_{0}$. Two significant wave heights, $H_{s}=1$ and $H_{s}=3 \mathrm{~m}$, are also utilized. Due to the constraints, the performance is similar to the one obtained in the unconstrained case only for short waves (small period), while it shows a peak for medium waves and it decreases again for longer waves, as theorized by Budal [4]. The suboptimal controller performs quite closely to MPC for short and long waves. It is less efficient, however, around the peak. Note that, unlike the unconstrained case, the bandwidth does not seem to affect the performance for the constrained case, although it affects the relative efficiency compared with MPC, which offers better results for narrowbanded waves, $\lambda=5$, most likely due to better predictions of the excitation force [17]. Note that for bigger waves, $H_{s}=3 \mathrm{~m}$, the trend of the power capture with wave 

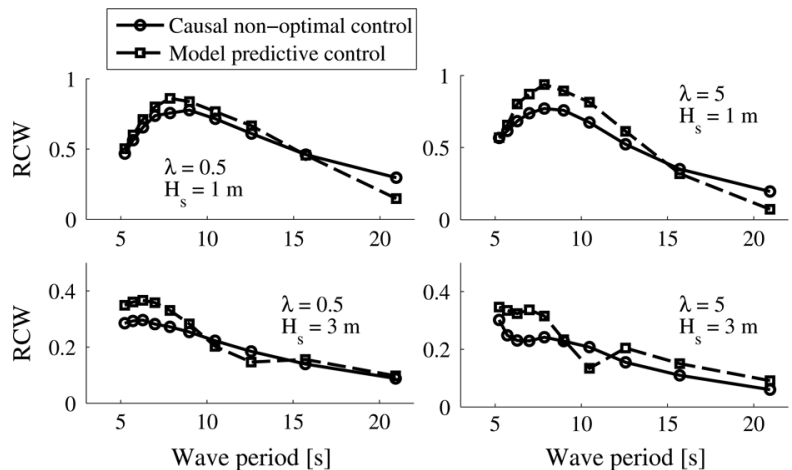

(a)
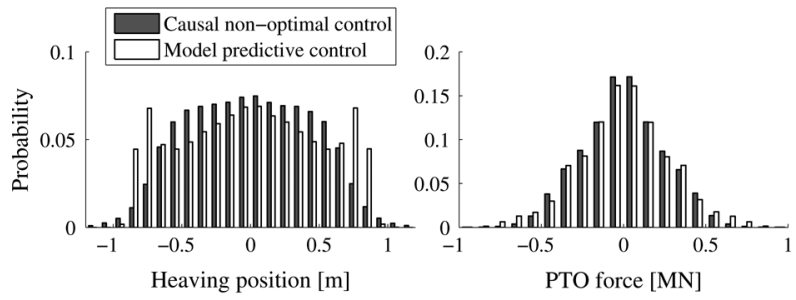

(b)

Fig. 13. Performance of proposed nonoptimal causal control, compared with optimal MPC. (a) RCW. (b) Distribution of heaving excursion and PTO force.

period is similar, but the peak moves towards smaller-period waves.

An important point that emerges from Fig. 13(a) is that, for very long waves, the suboptimal controller seems to outperform MPC. The reason behind this unexpected behavior may be found in the way MPC is specified, which depends on many parameters (optimization algorithm, future horizon, specification of functional, etc.) whose choice is not always straightforward, and our choice is probably not optimal enough for those waves. Such a behavior highlights one of the main benefits of the proposed controller, that although suboptimal, requires tuning of a single parameter which has intuitive physical meaning $(1 / H)$, representing the modulation of the magnitude of the velocity with respect to the excitation force.

A further advantage of the proposed controller is the linearity achieved between excitation force, velocity, position, and PTO force. Motion and force have the same harmonic behavior as the incident wave, with no abrupt variations that may adversely affect the survivability of the system components. The probability distribution of position [Fig. 13(b)] shows how MPC keeps the system mostly close to the constraints, with higher velocities in moving from one extreme to the other. The proposed controller, on the other hand, imposes a smooth and harmonic oscillation in the system, so that the position is almost normally distributed around the zero. Such a behavior can also be observed, though to a much lesser extent, in the force. Note that, for both controllers, the position goes slightly beyond the imposed constraint of $0.8 \mathrm{~m}$, due to model uncertainties. This unwanted behavior occurs slightly more frequently with the suboptimal controller, obviously, but can be overcome by adopting a more conservative reference-generation strategy than in (17). A sample time-series simulation, that makes clearer such considerations, is shown in Fig. 14, where the evolution of the position and the required control force are plotted for the two controllers.
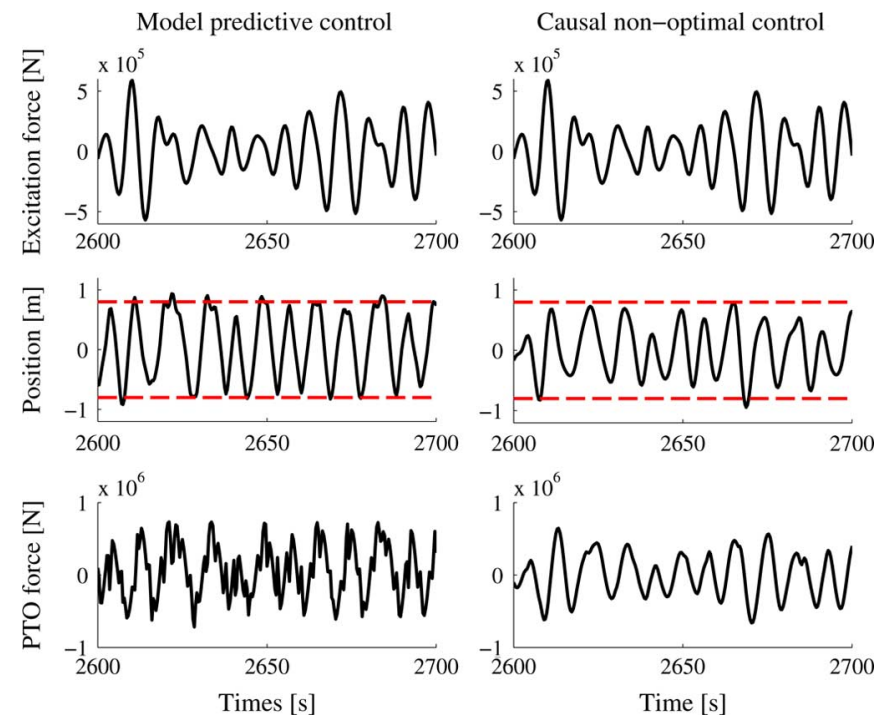

Fig. 14. Time series of a specific simulation. Input wave comes from an Ochi spectrum with $H_{s}=2 \mathrm{~m}, \omega_{0}=0.7 \mathrm{rad} / \mathrm{s}$, and $\lambda=1$.

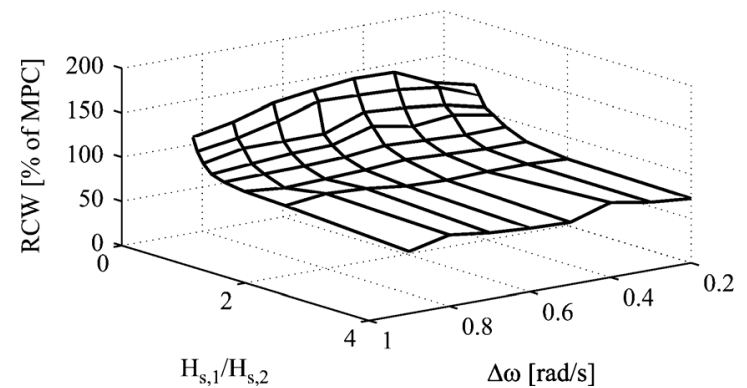

Fig. 15. Performance of causal control, as a percentage of MPC performance, with double peak spectra: $H_{s, 1} / H_{s, 2}$ is the relative energy between the two wave systems; $\Delta \omega$ is the frequency spacing.

The performance of the suboptimal controller, relatively to MPC, with double-peaked wave systems, is shown in Fig. 15. When the two peaks have a comparable size, the suboptimal controller outperforms MPC, particularly when $\Delta \omega$ is small. Such a behavior could be due to higher inaccuracies in the prediction of the excitation force, that is quite poor for multipeaked sea states [17].

The amount of reversed power required, in the presence of constraints, is shown in Fig. 16, where the ratio of reversed power to absorbed power is compared, for the proposed controller and MPC. Much less energy needs to be injected back from the PTO to the WEC, between $10 \%$ and $40 \%$ of the absorbed energy, than in the unconstrained case (100\% to $500 \%)$. Hydraulic or electrical generators can be designed to accomplish such levels of reversible energy flow, and the proposed controller can be optimized by taking into account inefficiencies in the two modes of operations, by acting on the constant $1 / H$.

\section{Performance With Real Wave Data}

The proposed controller is also validated with real wave data, provided by the Irish Marine Institute and coming from a data buoy in the Belmullet wave energy test site, off the West coast of Ireland. Three wave-elevation records of $30 \mathrm{~min}$, sampled at $1.28 \mathrm{~Hz}$, are selected. As shown in Fig. 17, a variety of sea 


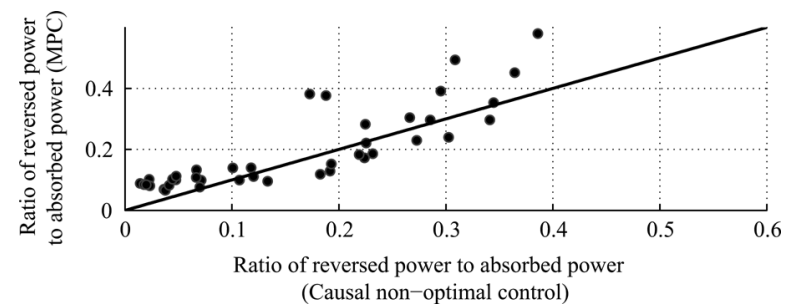

Fig. 16. Ratio of average reversed power (energy from PTO to system) to absorbed power (energy from system to PTO).
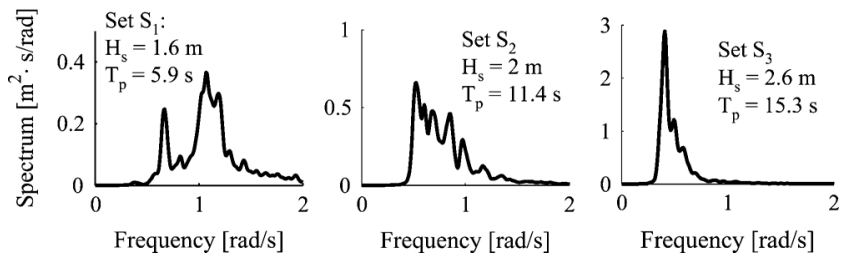

Fig. 17. Wave spectra of selected real wave data.

TABLE I

Performance With Real WaVe Data of Fig. 17

\begin{tabular}{|c|c||c|c|c|c|}
\hline \multirow{2}{*}{ Set } & \multicolumn{2}{|c||}{$P_{w}[\mathrm{~kW} / \mathrm{m}]$} & \multicolumn{2}{c|}{ Causal non-optimal control } & \multicolumn{2}{|c|}{ MPC } \\
& & $\bar{P}_{u}[\mathrm{~kW}]$ & $R C W$ & $\bar{P}_{u}[\mathrm{~kW}]$ & $R C W$ \\
\hline$S_{1}$ & 7.58 & 36.5 & 0.48 & 43.6 & 0.58 \\
$S_{2}$ & 17.0 & 57.2 & 0.34 & 62.7 & 0.37 \\
$S_{3}$ & 44.3 & 64.7 & 0.146 & 65.4 & 0.148 \\
\hline
\end{tabular}
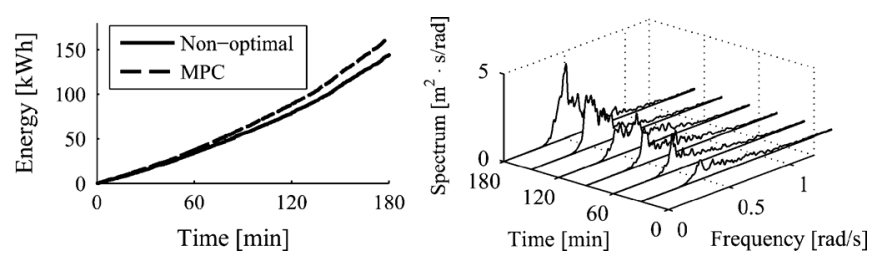

Fig. 18. Energy capture over a 3-h simulation with real wave data. The data were collected at Belmullet on October 1, 2010, 0:00 A.M.

conditions is covered, with the peak period going from 6 to $15 \mathrm{~s}$ and with different spectral bandwidth.

The performance of the proposed controller and of MPC is evaluated in the same conditions utilized for the simulated waves, in Section IV-C. Table I summarizes the results, by showing the average power absorption and the RCW with each of the records selected. In line with what is expected based on the general results of Fig. 13(a), the nonoptimal proposed controller performs almost as well as MPC.

The energy absorption over a 3-h simulation in real waves is also shown, in Fig. 18. Although real waves are nonstationary and the spectral distribution changes in time, the proposed controller maintains its performance, consistently with MPC.

\section{E. Sensitivity To Parameter Variations}

In wave energy, the typical operation of a device involves wide motions. Therefore, the typical assumptions of linearity, on which the model of Section II is based, may not be valid. The model is similar to a damped mechanical oscillator, with relatively small damping, and it makes sense to apply variations to one of the main terms that define the system, which is the hydrostatic stiffness $K_{s}$. Relative variations ranging from $\Delta K=-50 \%$ to $\Delta K=+50 \%$ are applied, resulting in a
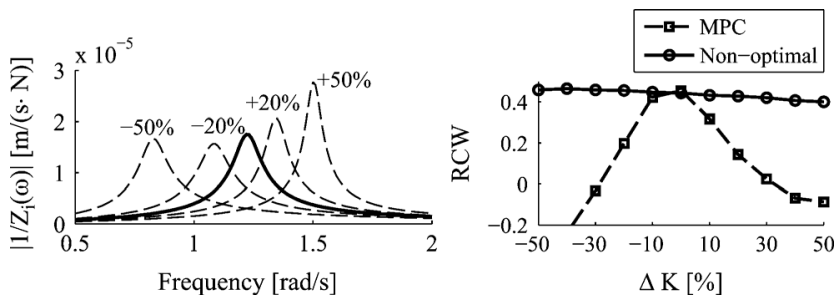

Fig. 19. Performance of the proposed nonoptimal controller against MPC, in the case of variations of the buoyancy coefficient $\Delta K$.

change in the dynamics, as shown in Fig. 19. A large variation is verified around the resonance frequency of the system, in particular.

A robustness analysis is carried out with the WEC being excited by irregular waves centered at a frequency of $1.25 \mathrm{rad} / \mathrm{s}$ (resonance frequency of the model). Fig. 19 shows how the $\mathrm{RCW}$ obtained with the proposed controller and with MPC is affected by parametric variations in such a wave condition. While MPC is quite robust for variations up to $\Delta K= \pm 10 \%$, the robustness of the proposed controller is clearly superior.

This is due to the fact that the reference velocity produced by the proposed controller, in the constrained region, does not depend on the model of the system, while MPC needs to solve an optimization based on such a model.

\section{CONCLUSION}

A novel strategy for the real-time control of bottom-referenced oscillating WECs has been proposed. The controller sets the velocity of the oscillator to be in phase with the excitation force and with an amplitude proportional to the wave force. The ratio between the magnitudes of velocity and excitation force can be tuned in real-time for best performance and constraints handling. While results were only shown for a bottom-referenced body, there is scope for generalizing the approach at least to self-referenced oscillating systems [35].

Based on a harmonic, nonstationary assumption about the wave excitation force, the proposed controller is suboptimal in real waves. However, it allows the achievement of levels of power capture close to MPC in most sea states, particularly in narrowbanded waves, without the need of predictions and of the solution of an optimization problem in real time. At the same time, the calculation of the reference velocity does not rely heavily on the model of the WEC, resulting in a superior robustness to model uncertainties. In addition, unlike MPC, the resulting motions and forces are linear with respect to the excitation force, giving velocities and forces that follow the same harmonic behavior as waves, with benefits in terms of long-term survivability of the WEC equipment. The effectiveness and computational simplicity make the proposed controller ideal for real-time applications.

While ideal efficiency in the bidirectional energy flow of the PTO mechanism was assumed, it was highlighted how the nature of the controller allows the possibility of including the efficiency information in the adaptive strategy of the high-level control. Also, the structure of the low-level control loop allows explicit addressing of the robustness to model uncertainties. Future work will go in such directions. 


\section{ACKNOWLEDGMENT}

The authors would like to thank the Irish Marine Institute for providing the wave data from the Belmullet test site.

\section{REFERENCES}

[1] D. Halamay, T. Brekken, A. Simmons, and S. McArthur, "Reserve requirement impacts of large-scale integration of wind, solar and ocean wave power generation," IEEE Trans. Sustain. Energy, vol. 2, no. 3, pp. 321-328, Jul. 2011.

[2] F. Fusco and J. Ringwood, "Variability reduction through combination of wind and waves: A Irish case study," Energy, vol. 35, pp. 314-325, 2010.

[3] X. Yang, Y. Song, G. Wang, and M. Wang, "A comprehensive review on the development of sustainable energy strategy and implementation in China," IEEE Trans. Sustain. Energy, vol. 1, no. 2, pp. 57-65, Jul. 2010 .

[4] J. Falnes, Ocean Waves and Oscillating Systems. Cambridge, U.K.: Cambridge Univ. Press, 2002.

[5] J. Falnes, "A review of wave-energy extraction," Marine Structures, vol. 20, pp. 285-201, 2007.

[6] F. Fusco and J. Ringwood, "A study of the prediction requirements in real-time control of wave energy converters," IEEE Trans. Sustain. Energy, vol. 3, no. 1, pp. 176-184, Jan. 2012.

[7] A. Babarit and A. Clement, "Optimal latching control of a wave energy device in regular and irregular waves," Appl. Ocean Res., vol. 28, pp. 77-91, 2006.

[8] K. Fuat, "Time domain prediction of power absorption from ocean waves with latching control," Renew. Energy, vol. 35, no. 2, pp. 423-434, 2010.

[9] J. J. Candido and P. A. P. S. Justino, "Modelling, control and pontryagin maximum principle for a two-body wave energy device," Renew. Energy, vol. 36, no. 5, pp. 1545-1557, 2011

[10] G. Bacelli, J.-C. Gilloteaux, and J. Ringwood, "A predictive controller for a heaving buoy producing potable water," in Proc. Eur. Control Conf. (ECC 2009), Budapest, Hungary, 2009, pp. 3755-3760.

[11] J. Cretel, A. W. Lewis, G. Lightbody, and G. P. Thomas, "An application of model predictive control to a wave energy point absorber," in Proc. IFAC Conf. Control Methodologies and Technology for Energy Efficiency (CMTEE 2010), Portugal, 2010.

[12] J. Hals, J. Falnes, and T. Moan, "Constrained optimal control of a heaving buoy wave-energy converter," J. Offshore Mechanics Arctic Eng., vol. 133, pp. 011401-1-011401-15, 2011.

[13] G. Bacelli, J. Ringwood, and J.-C. Gilloteaux, "A control system for a self-reacting point absorber subject to constraints," in Proc. 18th IFAC World Congress, Milano, Italy, 2011.

[14] M. P. Shoen, J. Hals, and T. Moan, "Wave prediction and robust control of heaving wave energy devices for irregular waves," IEEE Trans. Energy Convers., vol. 26, no. 2, pp. 627-638, Jun. 2011

[15] J. Cretel, A. W. Lewis, G. Thomas, and G. Lightbody, "A critical assessment of latching as a control strategy for wave-energy point absorbers," in Proc. 31st Int. Offshore and Polar Engineering Conf., Maui, HI, 2011.

[16] A. Falcao, P. Justino, J. Henriques, and J. Andre, "Reactive versus latching phase control of a two-body heaving wave energy converter," in Proc. 10th Eur. Control Conf. (ECC), Budapest, Hungary, 2009.

[17] F. Fusco and J. Ringwood, "Short-term wave forecasting for real-time control of wave energy converters," IEEE Trans. Sustain. Energy, vol. 1, no. 2, pp. 99-106, Jul. 2010.

[18] D. O'Sullivan, J. Griffiths, M. G. Egan, and A. W. Lewis, "Development of an electrical power take off system for a sea-test scaled offshore wave energy device," Renew. Energy, vol. 36, no. 4, pp. 1236-1244, 2011.

[19] R. Costello, J. V. Ringwood, and J. Weber, "Comparison of two alternative hydraulic PTO concepts for wave energy conversion," in Proc. 9th Eur. Wave and Tidal Energy Conf. (EWTEC), Southampton, U.K., 2011.

[20] E. Tedeschi, M. Carraro, M. Molinas, and P. Mattavelli, "Effect of control strategies and power take-off efficiency on the power capture from sea waves," IEEE Trans. Energy Convers., vol. 26, no. 4, pp. 1088-1098, Dec. 2011.
[21] P. Ricci, J. Lopez, M. Santos, P. Ruiz-Minguela, J. L. Villate, F. Salcedo, and A. F. O. Falcal, "Control strategies for a wave energy converter connected to a hydraulic power take-off," IET Renew. Power Generation, vol. 5, no. 3, pp. 1236-1244, 2011.

[22] Wamit. ver. 6.4, WAMIT Inc., MA, 2008.

[23] T. Perez and T. I. Fossen, "Time- vs. frequency-domain identification of parametric radiation force models for marine structures at zero speed," Modeling, Identification and Control, vol. 29, pp. 1-19, 2008.

[24] F. Fusco and J. Ringwood, "Suboptimal causal reactive control of wave energy converters using a second order system model," in Proc. Int Society of Offshore and Polar Eng. (ISOPE), Maui, HI, 2011.

[25] B. Quine, J. Uhlmann, and H. Durrant-Whyte, "Implicit jacobians for linearised state estimation in nonlinear systems," in Proc. Amer. Control Conf., Jun. 1995, pp. 1645-1646.

[26] K. Budal and J. Falnes, "The norwegian wave-power buoy project," in Proc. 2nd Int. Symp. Wave Energy Utillization, Trondheim, Jun. 1982, pp. 323-344.

[27] B. Boashash, "Estimating and interpreting the instantaneous frequency of a signal. II. Algorithms and applications," Proc. IEEE, vol. 80, no. 4, pp. 540-568, Apr. 1992.

[28] B. Boashash, "Estimating and interpreting the instantaneous frequency of a signal. I. Fundamentals," Proc. IEEE, vol. 80, no. 4, pp. 520-538, Apr. 1992.

[29] J. H. K. Shek, D. E. Macpherson, and J. Xiang, "Reaction force control of a linear electrical generator for direct drive wave energy conversion," IET Renew. Power Generation, vol. 1, pp. 17-24, 2007.

[30] F. Wu, X. P. Zhang, P. Ju, and M. J. H. Sterling, "Optimal control for aws-based wave energy conversion system," IEEE Trans. Power Syst., vol. 24, no. 4, pp. 1747-1755, Nov. 2009.

[31] M. Morari and Z. Evanghelos, Robust Process Control. Englewood Cliffs, NJ: Prentice-Hall, 1989.

[32] J. Sa de costa, P. Beirao, and D. Valerio, "Internal model control applied to the archimedes wave swing," in Proc. 16th Int. Conf. Control Systems and Computer Science (CSCS), Bucharest, Romania, 2007.

[33] M. K. Ochi, Ocean Waves: The Stochastic Approach. Cambridge, U.K.: Cambridge Univ. Press, 1998.

[34] J. Figwer, "A new method of random time-series simulation," Simulation Practise and Theory, vol. 5, pp. 217-324, 1997.

[35] J. Falnes, "Wave-energy conversion through relative motion between two single-mode oscillating bodies," Trans. ASME, J. Offshore Mechanics Arctic Eng., vol. 121, pp. 32-38, 1999.

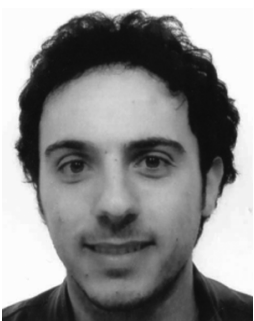

Francesco Fusco (S'09) received the Master degree in industrial automation engineering from Universita' Politecnica delle Marche (UNIVPM), Ancona, Italy, in 2008. From 2008 to 2011, he was a Ph.D. student at the Center for Ocean Energy Research (COER) of the National University of Ireland Maynooth (NUIM)

From 2008 to 2011 , he also worked as occasional lecturer at the COER. He is currently a postdoctoral researcher at the IBM Smart City Research Centre of Dublin. He is a Chartered Engineer in Italy.

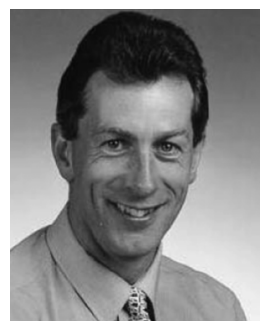

John V. Ringwood (M'87-SM'97) received the Diploma in electrical engineering from Dublin Institute of Technology and the Ph.D. degree in control systems from Strathclyde University, Scotland, in 1981 and 1985, respectively.

$\mathrm{He}$ is currently Professor of Electronic Engineering with the National University of Ireland (NUI), Maynooth, and Associate Dean for Engineering in the Faculty of Science and Engineering. $\mathrm{He}$ was Head of the Electronic Engineering Department at NUI Maynooth from 2000 until 2005, developing the Department from a greenfield site. His research interests cover time series modeling, wave energy, control of plasma processes, and biomedical engineering. He is a Chartered Engineer and a Fellow of the Institution of Engineers of Ireland. 